\title{
Direct search for light gluinos
}

\section{NA48 Collaboration}

V. Fanti ${ }^{\text {a }}$, A. Lai ${ }^{\text {a }}$, L. Musa ${ }^{\mathrm{a}, 1}$, D. Marras ${ }^{\text {a }}$, A. Nappi ${ }^{\text {a }}$, B. Hay ${ }^{\text {b }}$, R.W. Moore ${ }^{\text {b,2 }}$, K.N. Moore ${ }^{\text {b,2 }}$, D.J. Munday ${ }^{b}$, M.D. Needham ${ }^{\text {b }}$, M.A. Parker ${ }^{\text {b }}$, T.O. White ${ }^{b}$, S.A. Wotton ${ }^{\mathrm{b}}$, J. Andersen ${ }^{\mathrm{c}}$, G. Barr ${ }^{\mathrm{c}}$, G. Bocquet ${ }^{\mathrm{c}}$, J. Bremer ${ }^{\mathrm{c}}$, A. Ceccucci ${ }^{\mathrm{c}, 3}$, D. Cundy ${ }^{c}$, N. Doble ${ }^{\mathrm{c}}$, G. Fischer ${ }^{\mathrm{c}}$, W. Funk ${ }^{\mathrm{c}}$, L. Gatignon ${ }^{\mathrm{c}}$, A. Gianoli ${ }^{\mathrm{c}}$, A. Gonidec ${ }^{\text {c }}$, G. Govi ${ }^{\text {c }}$, P. Grafström ${ }^{\text {c }}$, W. Kubischta ${ }^{c}$, S. Luitz ${ }^{\text {c }}$, G. Kesseler ${ }^{\text {c }}$, J.P. Matheys ${ }^{c}$, A. Norton ${ }^{c}$, S. Palestini ${ }^{c, 3}$, B. Panzer-Steindel ${ }^{c}$, D. Schinzel ${ }^{c}$, H. Taureg ${ }^{c}$, M. Velasco ${ }^{\text {c,4 }}$, O. Vossnack ${ }^{c}$, H. Wahl ${ }^{c}$, G. Wirrer ${ }^{c}$, V. Kekelidze ${ }^{d}$, A. Mestvirishvili ${ }^{\mathrm{d}}$, I. Potrebenikov ${ }^{\mathrm{d}}$, G. Tatichvili ${ }^{\mathrm{d}}$, A. Tkatchev ${ }^{\mathrm{d}}$, A. Zinchenko ${ }^{\mathrm{d}}$, O. Boyle e , V.J. Martin e , I.G. Knowles ${ }^{\text {e }}$, H.L.C. Parsons ${ }^{\text {e }}$, P. Dalpiaz ${ }^{\text {f }}$, J. Duclos ${ }^{\text {f }}$, P.L. Frabetti ${ }^{\text {f }}$, M. Martini ${ }^{\mathrm{f}}$, F. Petrucci ${ }^{\mathrm{f}}$, M. Porcu ${ }^{\mathrm{f}}$, M. Savrié ${ }^{\mathrm{f}}$, A. Bizzeti ${ }^{\mathrm{g}}$, M. Calvetti ${ }^{\mathrm{g}}$, G. Graziani ${ }^{\mathrm{g}}$, E. Iacopini ${ }^{\mathrm{g}}$, M. Lenti ${ }^{\mathrm{g}}$, A. Michetti ${ }^{\mathrm{g}}$, H.G. Becker ${ }^{\mathrm{h}}$, H. Blümer h,1, P. Buchholz h,5, D. Coward h,6, C. Ebersberger h, H. Fox h, A. Kalter ${ }^{\text {h, K. Kleinknecht }}{ }^{\text {h }}$, U. Koch ${ }^{\text {h }}$, L. Köpke ${ }^{\text {h }, ~ B . ~ R e n k ~}{ }^{\text {h }}$, J. Scheidt ${ }^{\text {h }}$, J. Schmidt ${ }^{\mathrm{h}}$,V. Schönharting ${ }^{\mathrm{h}}$, I. Schué ${ }^{\mathrm{h}}$, R. Wilhelm ${ }^{\mathrm{h}}$, A. Winharting ${ }^{\mathrm{h}}$, M. Wittgen ${ }^{\text {h }}$ J.C. Chollet i , S. Crépé ${ }^{\mathrm{i}}$, L. Iconomidou-Fayard ${ }^{\mathrm{i}}$, L. Fayard ${ }^{\text {i }}$, J. Ocariz ${ }^{\text {i,7 }}$, G. Unal ${ }^{\text {i,D. Vattolo }}{ }^{\text {i }}$, I. Wingerter ${ }^{\text {i }}$, G. Anzivino ${ }^{\text {j, }}$ P. Cenci ${ }^{\mathrm{j}}$, P. Lubrano ${ }^{\mathrm{j}}$, M. Pepe ${ }^{\mathrm{j}}, \mathrm{B}$. Gorini ${ }^{\mathrm{k}, 1}$, P. Calafiura ${ }^{\mathrm{k}}$, R. Carosi ${ }^{\mathrm{k}}$, C. Cerri ${ }^{k}$, M. Cirilli k , F. Costantini ${ }^{k}$,R. Fantechi ${ }^{\text {k}}$, S. Giudici ${ }^{k}$, I. Mannelli ${ }^{k}$, V. Marzulli k , G. Pierazzini ${ }^{k}$, M. Sozzi ${ }^{k}, J . B$. Cheze ${ }^{1}$, J. Cogan ${ }^{1}$, M. De Beer ${ }^{1}$, P. Debu ${ }^{1}$, A. Formica ${ }^{1}$,R. Granier-De-Cassagnac ${ }^{1}$, P. Hristov ${ }^{1,8}$, E. Mazzucato ${ }^{1}$, B. Peyaud ', S. Schanne ', R. Turlay ', B. Vallage ', I. Augustin ${ }^{\mathrm{m}}$, M. Bender ${ }^{\mathrm{m}}$, M. Holder ${ }^{\mathrm{m}}$, M. Ziolkowski ${ }^{\mathrm{m}}$, R. Arcidiacono ${ }^{\mathrm{n}}$, C. Biino ${ }^{\mathrm{n}}$, F. Marchetto ${ }^{\mathrm{n}}$, E. Menichetti ${ }^{n}$, J. Nassalski ${ }^{\circ}$, E. Rondio ${ }^{\circ}$, M. Szleper ${ }^{\circ}$, W. Wislicki ${ }^{\circ}$, S. Wronka ${ }^{\circ}$, H. Dibon ${ }^{p}$, M. Jeitler ${ }^{p}$, M. Markytan ${ }^{p}$, I. Mikulec ${ }^{p}$, G. Neuhofer ${ }^{p}$, M. Pernicka ${ }^{\mathrm{p}}$, A. Taurok ${ }^{\mathrm{p}}$

\footnotetext{
a Dipartimento di Fisica dell'Università e Sezione dell'INFN di Cagliari, I-09100 Cagliari, Italy ${ }^{\mathrm{b}}$ Cavendish Laboratory, University of Cambridge, Cambridge, CB3 OHE, UK ${ }^{9}$ ${ }^{\mathrm{c}}$ CERN, CH-1211 Geneva 23, Switzerland

d Joint Institute for Nuclear Research, Dubna, Russian Federation
} 


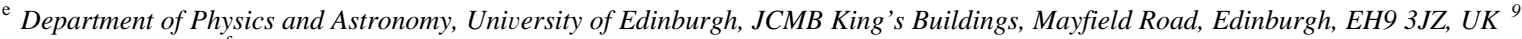

${ }_{\mathrm{f}}$ Dipartimento di Fisica dell'Università e Sezione dell'INFN di Ferrara, I-44100 Ferrara, Italy

g Dipartimento di Fisica dell'Università e Sezione dell'INFN di Firenze, I-50125 Firenze, Italy

${ }^{\mathrm{h}}$ Institut für Physik, Universität Mainz, D-55099 Mainz, Germany ${ }^{10}$

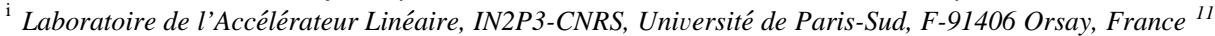

${ }^{j}$ Dipartimento di Fisica dell'Università e Sezione dell'INFN di Perugia, I-06100 Perugia, Italy

${ }^{\mathrm{k}}$ Dipartimento di Fisica, Scuola Normale Superiore e Sezione INFN di Pisa, I-56100 Pisa, Italy

${ }^{1}$ DSM / DAPNIA - CEA Saclay, F-91191 Gif-sur-Yvette, France

${ }^{\mathrm{m}}$ Fachbereich Physik, Universität Siegen, D-57068 Siegen, Germany ${ }^{12}$

${ }^{\mathrm{n}}$ Dipartimento di Fisica Sperimentale dell'Università e Sezione dell'INFN di Torino, I-10125 Torino, Italy

${ }^{\circ}$ Soltan Institute for Nuclear Studies, Laboratory for High Energy Physics, PL-00-681 Warsaw, Poland ${ }^{13}$

p Österreichische Akademie der Wissenschaften, Institut für Hochenergiephysik, A-1050 Wien, Austria
}

Received 28 October 1998

Editor: K. Winter

\begin{abstract}
We present the results for a direct search for light gluinos through the appearance of $\eta \rightarrow 3 \pi^{0}$ with high transverse momentum in the vacuum tank of the NA48 experiment at CERN. We find one event within a lifetime range of $10^{-9}-10^{-3}$ $\mathrm{s}$ and another one between $10^{-10}-10^{-9} \mathrm{~s}$. Both events are consistent with the expected background from neutrons in the beam, produced by $450 \mathrm{GeV}$ protons impinging on the Be targets, which interact with the residual air in the tank. From these data we give limits on the production of the hypothetical $g \tilde{g}$ bound state, the $\mathrm{R}^{0}$ hadron, and its $\mathrm{R}^{0} \rightarrow \eta \tilde{\gamma}$ decay in the $\mathrm{R}^{0}$ mass range between 1 and $5 \mathrm{GeV}$. (C) 1999 Elsevier Science B.V. All rights reserved.
\end{abstract}

\footnotetext{
${ }^{1}$ Present address: CERN, CH-1211 Geneva 23, Switzerland.

${ }^{2}$ Present address: Physics-Astronomy Building, Michigan State University, East Lansing, MI 48824, USA.

${ }^{3}$ Permanent address: Dipartimento di Fisica Sperimentale dell'Università e Sezione dell'INFN di Torino, I-10125 Torino, Italy.

${ }^{4}$ Corresponding author. E-mail: mayda.velasco@cern.ch

${ }^{5}$ Present address: Institut für Physik, Universität Dortmund, D-44221 Dortmund, Germany.

${ }^{6}$ Present address: SLAC, Stanford, CA 94309, USA.

${ }^{7}$ Permanent address: Departamento de Fíisica, Universidad de los Andes, Mérida 5101-A, Venezuela.

${ }^{8}$ Permanent address: Joint Institute for Nuclear Research, Dubna, Russian Federation.

${ }^{9}$ Funded by the UK Particle Physics and Astronomy Research Council.

${ }^{10}$ Funded by the German Federal Minister for Research and Technology (BMBF) under contract 7MZ18P(4)-TP2.

${ }^{11}$ Funded by Institut National de Physique des Particules et de Physique Nucléaire (IN2P3), France.

${ }^{12}$ Funded by the German Federal Minister for Research and Technology (BMBF) under contract 056SI74.

${ }^{13}$ Funded by the Committee for Scientific Research (KBN), grant 2 P03B07615.
}

Recent theoretical work [1,2] has proposed a class of supersymmetric models in which the gluino $(\tilde{g})$ and the photino $(\tilde{\gamma})$ are expected to have small masses and the photino is stable and an ideal candidate for dark matter. In such models there is a hypothetical spin-1/2 gluon-gluino ( $g \tilde{g}$ ) bound state, the $\mathrm{R}^{0}$ hadron. This strongly interacting particle is expected to have a mass of a few $\mathrm{GeV}$ and a lifetime between $10^{-10}$ and $10^{-6} \mathrm{~s}$. For these reasons the NA48 experiment [3] (see Fig. 1), designed to measure the $\mathrm{CP}$ violation parameter $\mathfrak{R}\left(\epsilon^{\prime} / \epsilon\right)$ using high intensity $\mathrm{K}_{\mathrm{L}}$ and $\mathrm{K}_{\mathrm{S}}$ beams, is a suitable experiment to look for $\mathrm{R}^{0}$, $\mathrm{s}$ produced by a $450 \mathrm{GeV}$ proton beam impinging on a $\mathrm{Be}$ target.

We have searched for $\mathrm{R}^{0} \rightarrow \eta \tilde{\gamma}$ through the appearance of $\eta \rightarrow 3 \pi^{0}$ with high transverse momentum in the decay volume of this experiment, under the assumption that the $\tilde{\gamma}$ is not detectable. Neutral kaons do not decay into $\eta$ 's because they are 50 $\mathrm{MeV}$ heavier, therefore $\eta$ 's are not expected to be found in decay volume of this experiment. The data 


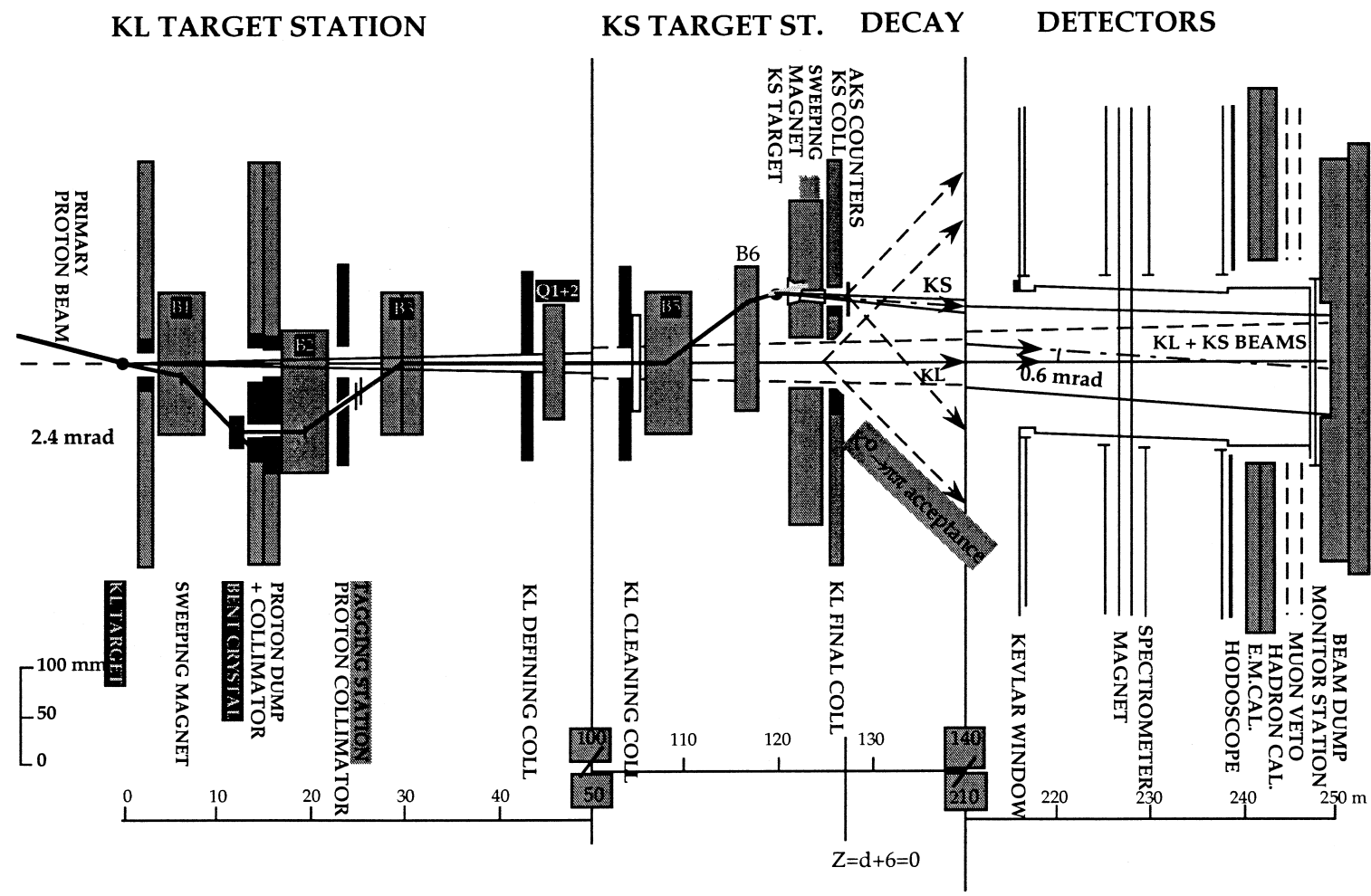

Fig. 1. Schematic drawing of the NA48 beamline and detector. In this detector photons are identified with a high resolution liquid krypton electromagnetic calorimeter. Extra photon activity is detected by a set of anti-counters located along the vacuum tank. Also shown is the charged spectrometer consisting of a magnet and wire chamber planes, complemented by a muon veto system, a hadronic calorimeter, and hodoscope triggering planes. See text for the definition of the longitudinal vertex position, $d$.

were collected in about 3 weeks of data taking during 1997.

As shown in Fig. 1, the NA48 experiment has two nearly collinear $\mathrm{K}_{\mathrm{S}}$ and $\mathrm{K}_{\mathrm{L}}$ beams which operate concurrently [4]. The beams are produced from 1.1 $\times 10^{12}$ and $3.4 \times 10^{7}$ protons impinging on the 40 cm long $\mathrm{K}_{\mathrm{L}}$ and $\mathrm{K}_{\mathrm{S}}$ Be targets, respectively, every $14.4 \mathrm{~s}$ in a burst that is $2.4 \mathrm{~s}$ long. Decays occurring in the $\mathrm{K}_{\mathrm{S}}$ and $\mathrm{K}_{\mathrm{L}}$ beamline are distinguished by a tagging scintillator hodoscope which is positioned in the proton beam producing the $\mathrm{K}_{\mathrm{S}}$ beam by measuring the time of flight between the tagging scintillator hodoscope and the main detector. This dual beamline design offers a wide lifetime range $\left(10^{-10}-10^{-3} \mathrm{~s}\right)$ in the $\mathrm{R}^{0}$ search.

A dedicated trigger derived from the $\epsilon^{\prime} / \epsilon$ neutral trigger, based on the liquid krypton electromagnetic calorimeter ( $\mathrm{LKr}$ ) [5] information, was implemented in order to select $3 \pi^{0}$ events with high transverse momentum (high- $P_{\mathrm{T}}$ ). The complete 'neutral' trigger system is described in Ref. [6]. The high- $P_{\mathrm{T}}$ trigger decision was based on the calculated total electromagnetic energy $E_{\mathrm{LKr}}$, the first moment of the energy $m_{1}$, the energy center-of-gravity COG, and the number of clusters in each projection. These quantities are calculated from:

$E_{\mathrm{LKr}}=0.5 \times\left(m_{0 x}+m_{0 y}\right)$,

$m_{1}=\sqrt{m_{1 x}^{2}+m_{1 y}^{2}}$,

$\mathrm{COG}=m_{1} / E_{\mathrm{LKr}}$,

where $m_{0 x}=\sum_{i} E_{i}, m_{0 y}=\sum_{j} E_{j}, m_{1 x}=\sum_{i} x_{i} E_{i}, m_{1 y}$ $=\sum_{j} y_{j} E_{j}$, are the calculated moments in each projection, and the $i$ and $j$ indices denote summation over energies and position of the vertical ( $x$-projection) and horizontal ( $y$-projection) strips of the $\mathrm{LKr}$ calorimeter. The point $(x=0, y=0)$ is defined at the 
center of the calorimeter. The resulting high- $P_{\mathrm{T}}$ trigger had a high background rejection power with losses less than $25 \%$ of the geometrically accepted signal. This was achieved by requiring $E_{\mathrm{LKr}} \geq 40$ $\mathrm{GeV}, m_{1} \geq 1500 \mathrm{GeV} \mathrm{cm}, \mathrm{COG} \geq 20 \mathrm{~cm}$, and four or more distinguishable clusters in at least one of the two projections. The COG requirement limits the transverse momentum to more than $0.15 \mathrm{GeV}$ for $\eta \mathrm{s}$ of momentum greater then $75 \mathrm{GeV}$, while the first moment requirement rejects backgrounds from $\mathrm{K}_{\mathrm{L}}$ $\rightarrow 3 \pi^{0}$ 's, where some of the photons escape detection. The largest measured loss $(15 \%)$ for fully contained events is due to the overlapping of clusters in both projections. For the final trigger to be issued, the neutral trigger signal required to be in anti-coincidence with the muon veto and the ring-shape array of photon detectors appearing as "anti-counters" in Fig. 1. The high- $P_{\mathrm{T}}$ trigger was downscaled by a factor of two. The resulting trigger rate was below 100 triggers/burst out of a total of 13,000 triggers / burst handled by the data acquisition system during the data taking using $1.5 \times 10^{12}$ protons / burst on the $\mathrm{K}_{\mathrm{L}}$ target.

The six photons in an event are used to reconstruct three $\pi^{0}$ 's that have to come from a common vertex. The photons must have energies above 2 $\mathrm{GeV}$, a time difference between them which is smaller than $1.5 \mathrm{~ns}$, to be within the defined $\mathrm{LKr}$ fiducial volume, and to have no track in the drift chambers. In addition, it is required that there is no activity in the hadronic calorimeter and that the energy of the $\eta$ 's is greater than $95 \mathrm{GeV}$. The reconstructed $\pi^{0}$ masses, $m_{i}(d)$, are used as constraints in a fit to minimize the $\chi^{2}$ as a function of the longitudinal vertex position, $d$, without an assumption on the mass of the parent particle, that is, $\chi^{2}(d)=\sum_{i=1}^{3}\left(m_{\pi^{0}}-m_{i}(d)\right)^{2} / \sigma_{i}^{2}$. The typical error on the $\pi^{0}$ mass $\sigma_{i}$ is around $1.2 \mathrm{MeV}$. We will set a mass window $\pm 6 \mathrm{MeV}$ wide, which corresponds to approximately $\pm 3 \sigma$ for $3 \pi^{0}$ events. The $\chi^{2}(d)$ was required to be smaller than 8 , which corresponds to a confidence level (CL) larger than $98.2 \%$.

The masses of the selected high- $P_{\mathrm{T}}$ events are shown in Fig. 2 as a function of the best fit longitudinal vertex position. We find $152 \mathrm{~K}_{\mathrm{L}} \rightarrow 3 \pi^{0}$ events and $31 \eta \rightarrow 3 \pi^{0}$ events in the $d$-region between -300 and $9600 \mathrm{~m}$. The integrated beam corresponds to $1.2 \times 10^{17}$ and $2.1 \times 10^{12}$ protons impinging on

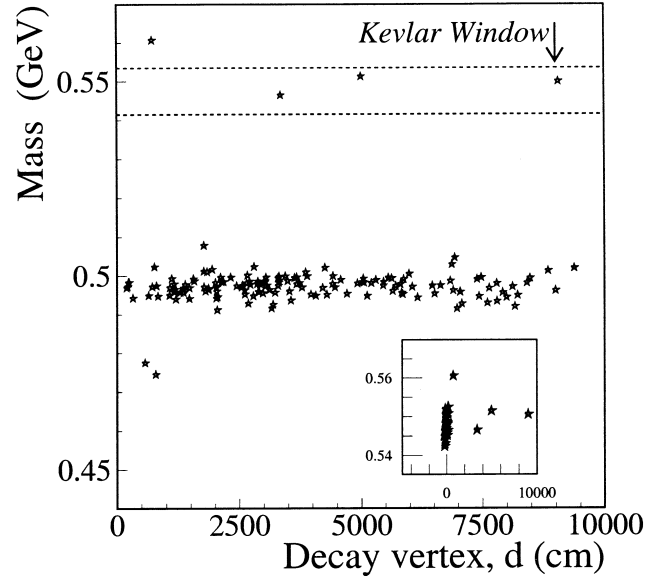

Fig. 2. Reconstructed high transverse momentum $\mathrm{K}_{\mathrm{L}}$ and $\eta$ particle decays into $3 \pi^{0}$ in the vacuum region. The two horizontal lines define the mass window for $\eta$ candidates. The inserted plot includes the $\eta$ events produced by beam interactions in elements of the beamline (see Fig. 3 and Fig. 4) before the allowed fiducial decay volume.

the $\mathrm{K}_{\mathrm{L}}$ and $\mathrm{K}_{\mathrm{S}}$ targets, respectively. The COG distribution for the $\mathrm{K}_{\mathrm{L}} \rightarrow 3 \pi^{0}$ events is consistent with simulations made for elastic and quasi-elastic interactions in the AKS and beam cleaning collimators shown in Fig. 3.

Since the $\eta$ has a very short lifetime, its decay vertex practically coincides with the position at which it was produced. Therefore, the expected $\mathrm{R}^{0}$ signature is an $\eta$ with high- $P_{\mathrm{T}}$ in the vacuum region right after the last collimators and the AKS counter. The fiducial region begins around $6 \mathrm{~m}$ downstream of the $\mathrm{K}_{\mathrm{S}}$ target, and ends at around $96 \mathrm{~m}$ downstream at the Kevlar window, see Fig. 1. The vertex resolution for $\eta \rightarrow 3 \pi^{0}$ events is about $70 \mathrm{~cm}$. Therefore, in order to reduce the background from $\eta$ 's produced in the collimators and the AKS counter, only events with a vertex which is at least $200 \mathrm{~cm}$ away from the AKS counter position were accepted. As shown in Fig. 2, there are three events that survive all the above cuts and they are in the mass window of \pm 6 $\mathrm{MeV}$. of the mass resolution for $3 \pi^{0}$ events. The vertex of the most downstream event is consistent with the position of the Kevlar window, and therefore is excluded from the analysis. The two remaining events are identified as one particle coming from the $\mathrm{K}_{\mathrm{L}}$ Be target and the other one from the $\mathrm{K}_{\mathrm{S}} \mathrm{Be}$ target, by comparing the time of the event as defined 


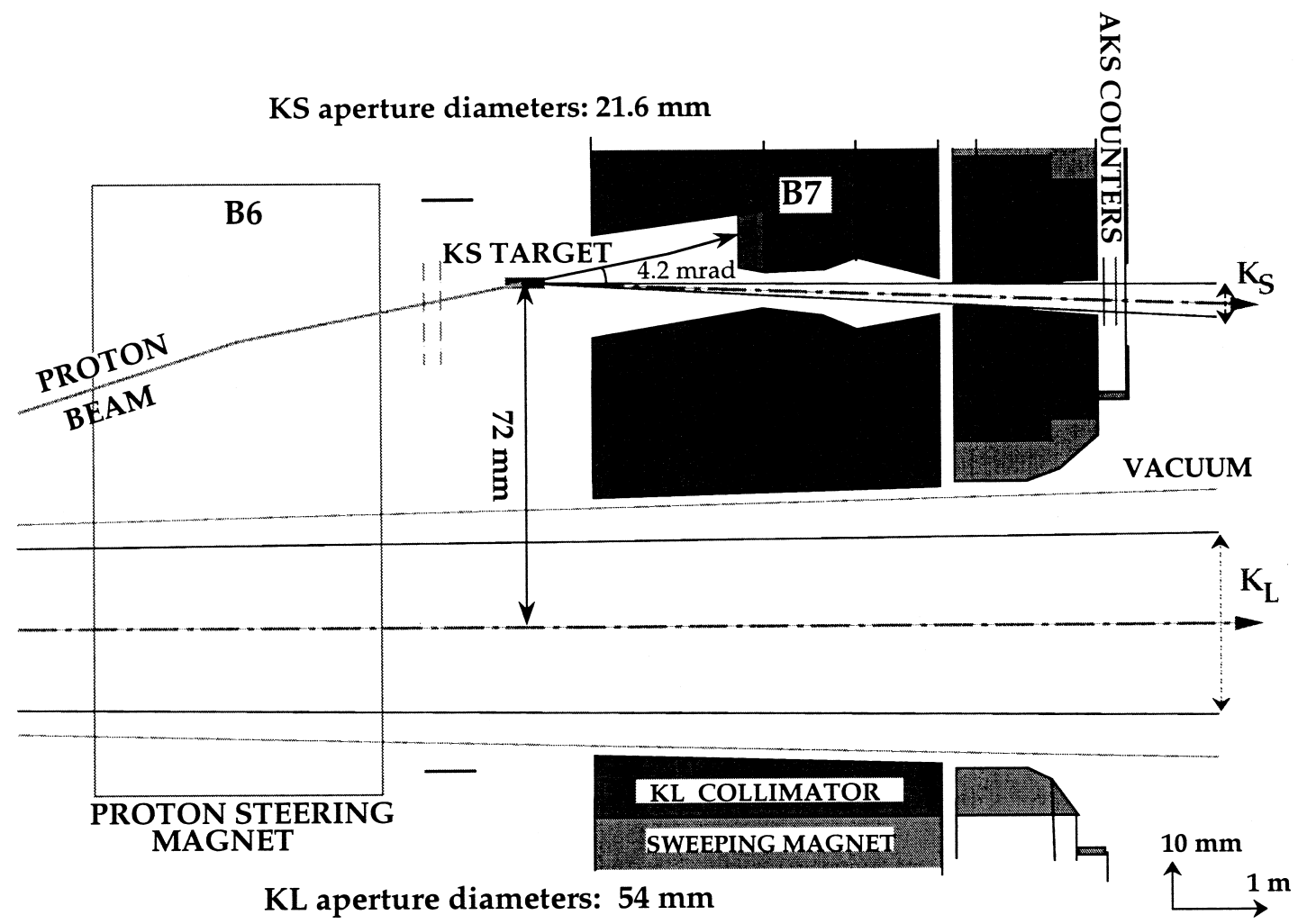

Fig. 3. Schematic drawing of the last set of beam collimators for the $K_{S}$ and $K_{L}$ beamline.

by the $\mathrm{LKr}$ system and the $\mathrm{K}_{\mathrm{S}}$ proton tagging system [7]. A time difference smaller than $1.5 \mathrm{~ns}$ is required for an association with the $\mathrm{K}_{\mathrm{S}}$ target. We assign one event produced in the $\mathrm{K}_{\mathrm{L}}$ beamline and the other one in the $\mathrm{K}_{\mathrm{S}}$ beamline. The tagging system has an efficiency greater than $99.9 \%$, but the rate of protons in the tagger is of the order of $30 \mathrm{MHz}$, which gives a probability greater than $10 \%$ of having an event from the $\mathrm{K}_{\mathrm{L}}$ beamline identified as coming from the $\mathrm{K}_{\mathrm{S}}$ due to an accidental coincidence.

The main background is due to diffractive neutron interactions in the remaining air in the $6-9 \times 10^{-5}$ mbar vacuum region. There are also about $10^{9}$ photons/burst coming from the $\mathrm{K}_{\mathrm{L}}$ beamline, but they do not contribute to the background because their mean energy is only $30 \mathrm{GeV}$. The expected mean energy for the neutrons in the $\mathrm{K}_{\mathrm{L}}$ and $\mathrm{K}_{\mathrm{S}}$ beamlines is around $190 \mathrm{GeV}$ and $100 \mathrm{GeV}$, and the expected rates are $2 \times 10^{8}$ and $1.5 \times 10^{4}$ per burst, respectively.
The background estimates are based on a special run taken with a charged $75 \mathrm{GeV}$ pion beam where $\pi^{-} \mathrm{N} \rightarrow \eta \mathrm{X}$ events were recorded, and where $\sigma\left(\pi^{-} \mathrm{N} \rightarrow \eta \mathrm{X}\right)$ was measured from eight hours of data taking in which $10^{7}$ pions/burst hit a $6 \mathrm{~cm}$ thick $\mathrm{CH}_{2}$-target at the nominal SPS cycle time. In these data only $\eta \rightarrow 3 \pi^{0}$ events with $\eta$ energies above $95 \mathrm{GeV}$ had trigger requirements similar to those of the high- $P_{\mathrm{T}} 3 \pi^{0}$ trigger. For this reason, as already mentioned a minimum energy requirement of $95 \mathrm{GeV}$ in the analysis was applied for the events shown in Fig. 2. However, we do not see additional high- $P_{\mathrm{T}} \eta$ events if this requirement is relaxed.

The estimates for high- $P_{\mathrm{T}} \eta$ production in interactions of neutrons in the vacuum tank are found from the ratios of cross sections $\sigma\left(\pi^{-} \mathrm{N} \rightarrow\right.$ $\eta \mathrm{X}) / \sigma(\mathrm{nN} \rightarrow \eta \mathrm{X})$. We find that our sample should contain about 0.4 and $1.0 \times\left(10^{-5}\right) \eta$ 's in the $\mathrm{K}_{\mathrm{L}}$ and the $\mathrm{K}_{\mathrm{S}}$ beamline, respectively. The background estimates for the $\mathrm{K}_{\mathrm{S}}$ beamline can be cross checked 
using the $\eta$ events produced in the collimators and the $2 \mathrm{~mm}$ Iridium crystal (AKS) [8]. The AKS is located around $6 \mathrm{~m}$ after the $\mathrm{K}_{\mathrm{S}}$ target, and is used to detect $\mathrm{K}_{\mathrm{S}}$ decaying before this point. As shown in the inserted plot in Fig. 2 and in more detail in Fig. 4, there are 31 events produced in the region of the AKS and collimators. According to the tagging system 13 of them are in the $K_{S}$ beamline which implies an expected background of $0.4 \times 10^{-5} \eta$ events produced by all particles in that beamline. This is consistent with the $\pi \mathrm{N}$ estimates.

As discussed above, there is a $10 \%$ probability that an event produced in the $\mathrm{K}_{\mathrm{L}}$ beamline is assigned to the $\mathrm{K}_{\mathrm{S}}$ instead, and a $40 \%$ probability of having an $\eta$ event in our data sample that was produced by neutrons in the $\mathrm{K}_{\mathrm{L}}$ beamline. As a consequence, the probability of having two $\mathrm{K}_{\mathrm{L}}$ events and that one of them is tagged as from the $\mathrm{K}_{\mathrm{S}}$ beamline is $1.2 \%$. Therefore, we conclude that all events, both in the $\mathrm{K}_{\mathrm{S}}$ and the $\mathrm{K}_{\mathrm{L}}$ beamline, are consistent with background expectation.

As shown in Fig. 5, the detector acceptance for $\eta \rightarrow 3 \pi^{0}(\mathrm{BR} \simeq 32 \%)$ events produced from $\mathrm{R}^{0} \rightarrow \eta \tilde{\gamma}$ decaying within the fiducial volume, shows a strong dependence on the mass ratio between the $\mathrm{R}^{0}$ and the $\tilde{\gamma}, r=m_{\mathrm{R}^{0}} / m_{\tilde{\gamma}}$. At long lifetimes and/or for $r-1 \gg m_{\eta} / m_{\tilde{\gamma}}$ the acceptance becomes almost in-

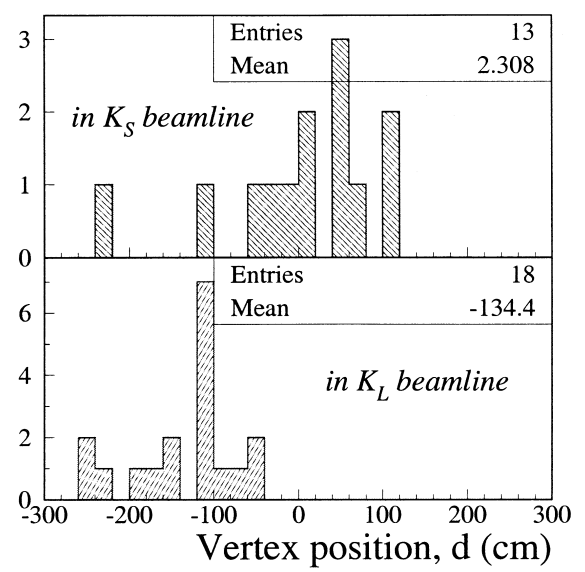

Fig. 4. Vertex distribution for $\eta$ particles produced in the beam collimators region, see Fig. 3. Based on the vertex position, we can conclude that most of the events coming from the $\mathrm{K}_{\mathrm{S}}$ beamline were produced at the AKS. As in the analysis, the zero vertex position is defined at the location of the AKS Iridium crystal.

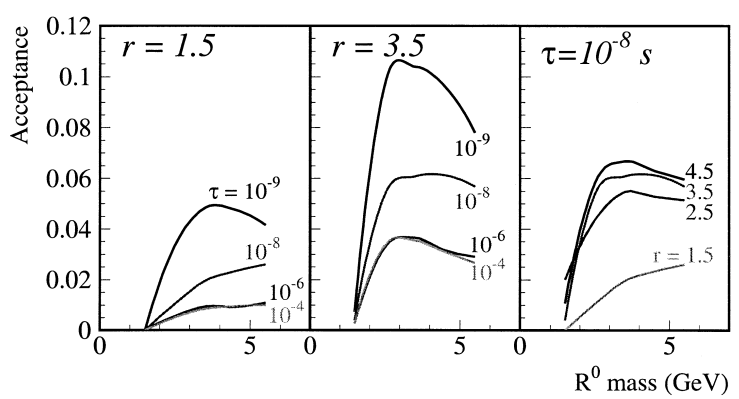

Fig. 5. Total selection efficiency for several values of the mass ratio $r=m_{\mathrm{R}^{0}} / m_{\tilde{\gamma}}$ and $\mathrm{R}^{0}$ lifetime as a function of the $\mathrm{R}^{0}$ mass.

dependent of $r$. We have assumed that the $\mathrm{R}^{0}$ energy spectrum is the same as that of $\Lambda$ production measured by this experiment [9]. The sensitivity to the energy spectrum is weak, and it is seen only at short lifetimes, where the sensitivity drops very quickly as a function of $\mathrm{R}^{0}$ mass.

The angular acceptance in the $\mathrm{K}_{\mathrm{L}}$ and the $\mathrm{K}_{\mathrm{S}}$ beamline is 0.15 and $0.375 \mathrm{mrad}$, respectively. The maximum difference in the angular acceptance between neutral kaons and $\pi^{ \pm}, \mathrm{K}^{ \pm}, \mathrm{p}, \overline{\mathrm{p}}$ was found using the results from Ref. [10] and used as an upper estimate of the expected difference in 'collimator' acceptance between $\mathrm{R}^{0}$ and neutral kaons. We concluded that the $\mathrm{R}^{0}$ 'collimator' acceptance will be smaller than that of kaons by about $4 \%$ and $23 \%$ in the $\mathrm{K}_{\mathrm{L}}$ and the $\mathrm{K}_{\mathrm{S}}$ beamline, respectively.

The expected interaction rate for $\mathrm{R}^{0} \mathrm{~N}$ is expected to be between $10 \%$ to $100 \%$ of the $\mathrm{pN}$ cross section [11]. This means that the ratio of the absorption
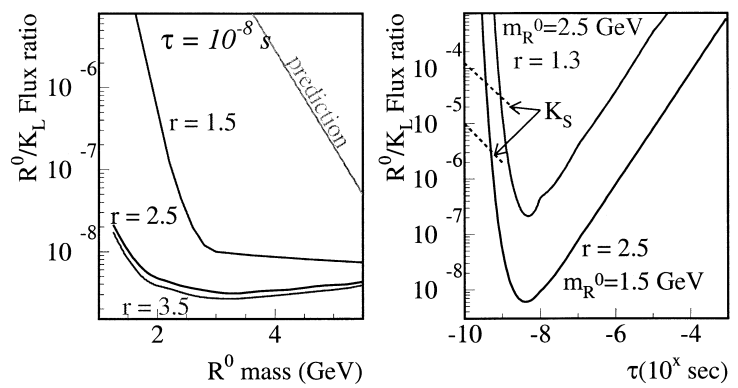

Fig. 6. Upper limits at $95 \%$ confidence level on the flux ratio of $\mathrm{R}^{0}$ and $\mathrm{K}_{\mathrm{L}}$ production in $\mathrm{p}^{+}-\mathrm{Be}$ interactions assuming a $100 \%$ branching ratio for this decay mode. A small improvement in the exclusion at small lifetime is obtained from $\mathrm{K}_{\mathrm{S}}$ events. 

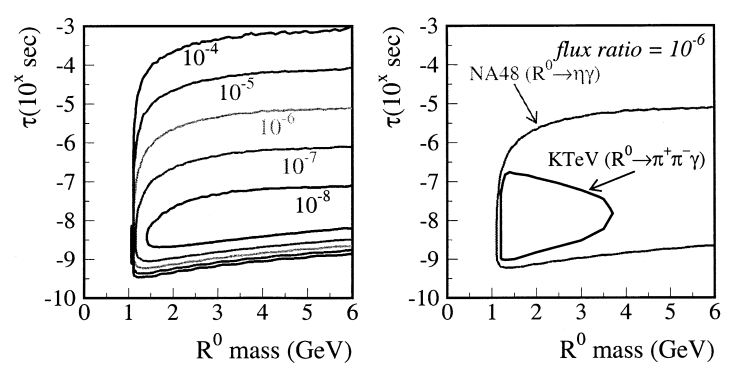

Fig. 7. Upper limits at $95 \% \mathrm{CL}$ on the flux ratio between $\mathrm{R}^{0}$ and $\mathrm{K}_{\mathrm{L}}$ production in $\mathrm{p}-\mathrm{Be}$ interactions for $r=2.2$. The second plot shows the $10^{-6}$ contours limits given by the analysis presented here and by the $\mathrm{KTeV}$ collaboration. In both cases a $100 \%$ branching ratio in the analysed decay mode $\mathrm{R}^{0} \rightarrow \eta \tilde{\gamma}$ was assumed.

probabilities in the Be target of $\mathrm{R}^{0}$ and kaons can be between 0.75 and 1 .

It is more conservative to not apply a background subtraction, but to evaluate the limits based on one signal event in each beam. In addition, it is assumed that the branching ratio of $\mathrm{R}^{0} \rightarrow \eta \tilde{\gamma}$ is equal to $100 \%$. After taking all the above in consideration and since most of our data comes from the $\mathrm{K}_{\mathrm{L}}$ beamline, we first consider the ration of $\mathrm{R}^{0}$ and $\mathrm{K}_{\mathrm{L}}$ fluxes. The resulting limits on the flux ratio between $\mathrm{R}^{0} \rightarrow \eta \tilde{\gamma}$ and $\mathrm{K}_{\mathrm{L}}$ production at a $95 \% \mathrm{CL}$ are shown in Fig. 6(a),(b). The fall-off in the sensitivity for small $\mathrm{R}^{0}$ mass on the left side of plot (a) is due to the loss in phase space for the $\eta$ to be produced, while the drop in the right side in (b) is due to the decrease in detected events as the lifetime increases.

The numbers of expected $\mathrm{K}_{\mathrm{L}}$ and $\mathrm{K}_{\mathrm{S}}$ at the exit of the last collimator are $2 \times 10^{7}$ and $2 \times 10^{2}$, respectively. This means that there are also enough protons in the $\mathrm{K}_{\mathrm{S}}$ target in order to improve the limits at low lifetimes. The $\mathrm{K}_{\mathrm{S}}$ data gives an upper bound of $10^{-5}$ and $10^{-4}$ for $r=2.5$ and $r=1.3$, respectively, based on one event.

These results can be summarized for $r=2.2$ by plotting the contours of the upper limits at 95\% CL. These are shown in Fig. 7 where they are compared with the best limit at $90 \%$ CL from the direct search for $\mathrm{R}^{0} \rightarrow \pi^{+} \pi^{-} \tilde{\gamma}$ by the KTeV Collaboration [12]. The two searches are complementary and not necessarily comparable because of the different decay modes. Both analyses show their results by setting the indicated branching ratio equal to $100 \%$. The two-body $\mathrm{R}^{0}$ decays are suppressed due to approximate $C$ invariance in SUSY QCD [2], while the three-body decays are not. The $\pi^{0}, \eta$ and $\mathrm{R}^{0}$ have $C=+1$, while $C=-1$ for photinos. Nevertheless, our limits on the $\mathrm{R}^{0} / \mathrm{K}_{\mathrm{L}}$ flux ratio are stringent on the $\mathrm{R}^{0}$ production even if the branching ratio of $\mathrm{R}^{0} \rightarrow \eta \tilde{\gamma}$ is of the order of $10^{-2}$.

Though $\mathrm{R}^{0}$ production cross sections are quite model dependent, and the theoretical uncertainties in the estimates of the $\mathrm{R}^{0}$ branching ratio into $\mathrm{R}^{0} \rightarrow \eta \tilde{\gamma}$ and the productions cross sections of $\mathrm{R}^{0}$ 's are rather large. Nevertheless, the available perturbative QCD calculations [13] imply that the $\mathrm{R}^{0} / \mathrm{K}_{\mathrm{L}}$ flux ratio goes as $0.14 \mathrm{e}^{-2.7 m_{\mathrm{R}^{0}}}$. This implies that $\mathrm{R}^{0} \mathrm{~S}$ with low mass are excluded by these results even if the branching ratio for $\mathrm{R}^{0} \rightarrow \eta \tilde{\gamma}$ is at the level of $1 \%$, as shown in Fig. 7 for an $\mathrm{R}^{0}$ lifetime of $10^{-8}$.

In conclusion, limits are given on the upper values for the $\mathrm{R}^{0} / \mathrm{K}_{\mathrm{L}}$ flux ratio in a region of $\mathrm{R}^{0}$ mass and lifetime between $1-5 \mathrm{GeV}$ and $10^{-10}-10^{-3} \mathrm{~s}$, respectively. As shown in Fig. 6, depending on the value for the branching ratio of $\mathrm{R}^{0} \rightarrow \eta \tilde{\gamma}$, the $95 \%$ $\mathrm{CL}$ on the upper value on the $\mathrm{R}^{0} / \mathrm{K}_{\mathrm{L}}$ flux ratio could be as low as $6 \times 10^{-9}$ for a $\mathrm{R}^{0}$ with a mass of $1.5 \mathrm{GeV}$, a lifetime of $6 \times 10^{-9} \mathrm{~s}$ and $r=2.5$.

\section{Acknowledgements}

We would like to thank the technical staff of the participating Laboratories, Universities and affiliated computing centres for their efforts in the construction of the NA48 detector, their help in the operation of the experiment, and in the processing of the data. The Cambridge and Edinburgh groups thank the UK Particle Physics and Astronomy Research Council for financial support.

\section{References}

[1] G. Farrar, Phys. Rev. D 51 (1995) 3904.

[2] G. Farrar, Phys. Rev. Lett. 76 (1996) 4111.

[3] NA48 Collaboration, G. Barr et al., Proposal for a precision Measurement of $\epsilon^{\prime} / \epsilon$ in CP Violating $\mathrm{K}^{0} \rightarrow 2 \pi$ Decays, CERN/SPSC/90-22, 1990.

[4] C. Binno, N. Doble, L. Gatignon, P. Grafstrom, H. Wahl, The simultaneous Long- and Short-lived neutral kaon beams for experiment NA48, CERN-SL-98-033 (EA). 
[5] NA48 Collaboration, G. Barr et al., Nucl. Instr. and Meth. A 370 (1996) 413.

[6] B. Gorini et al., IEEE Trans. Nucl. Sci. 45 (1998) 1771; G. Fischer et al., Presented to Vienna Wire Chamber Conference, 1998.

[7] T. Beier et al., Nucl. Instr. and Meth. A 360 (1995) 390.

[8] C. Biino, Workshop on Channelling and Other Coherent Crystal Effects at Relativistic Energies, Aarhus, Denmark, July, 1995.

[9] NA48 Collaboration, V. Fanti et al., A Measurement of the
Transversal Polarization of $\Lambda$-Hyperons Produced in Inelastic pN-Reactions at $450 \mathrm{GeV}$ Proton, submitted to Euro. Phys. Jour. C.

[10] H.W. Atherton et al., CERN Yellow Report 80-07, 1980.

[11] S. Nussinov, Phys. Rev. D 57 (1998) 7006.

[12] KTeV Collaboration, J. Adams, Phys. Rev. Lett. 79 (1997) 4083.

[13] S. Dawson, E. Eichten, C. Quigg, Phys. Rev. D 37 (1985) 1581; C. Quigg, private communication; S.V. Somalwar, private communication. 